\title{
3D synthetic aperture imaging using a virtual source element in the elevation plane
}

\author{
Nikolov, Svetoslav; Jensen, Jørgen Arendt
}

Published in:

Proceedings of IEEE Ultrasonics Symposium

Link to article, DOI:

10.1109/ULTSYM.2000.921659

Publication date:

2000

Document Version

Publisher's PDF, also known as Version of record

Link back to DTU Orbit

Citation (APA):

Nikolov, S., \& Jensen, J. A. (2000). 3D synthetic aperture imaging using a virtual source element in the elevation plane. In Proceedings of IEEE Ultrasonics Symposium (Vol. 2, pp. 1743-1747). IEEE.

https://doi.org/10.1109/ULTSYM.2000.921659

\section{General rights}

Copyright and moral rights for the publications made accessible in the public portal are retained by the authors and/or other copyright owners and it is a condition of accessing publications that users recognise and abide by the legal requirements associated with these rights.

- Users may download and print one copy of any publication from the public portal for the purpose of private study or research.

- You may not further distribute the material or use it for any profit-making activity or commercial gain

- You may freely distribute the URL identifying the publication in the public portal 


\title{
3D synthetic aperture imaging using a virtual source element in the elevation plane
}

\author{
Svetoslav Ivanov Nikolov and Jørgen Arendt Jensen \\ Center for Fast Ultrasound Imaging, Department of Information Technology, Build. 344, \\ Technical University of Denmark, DK-2800 Lyngby, Denmark
}

\begin{abstract}
The conventional scanning techniques are not directly extendable for 3D real-time imaging because of the time necessary to acquire one volume. Using a linear array and synthetic transmit aperture, the volume can be scanned plane by plane. Up to 1000 planes per second can be scanned for a typical scan depth of $15 \mathrm{~cm}$ and speed of sound of $1540 \mathrm{~m} / \mathrm{s}$. Only 70 to 90 planes must be acquired per volume, making this method suitable for real-time $3 \mathrm{D}$ imaging without compromising the image quality. The resolution in the azimuthal plane has the quality of a dynamically focused image in transmit and receive. However, the resolution in the elevation plane is determined by the fixed mechanical elevation focus.

This paper suggests to post-focus the RF lines from several adjacent planes in the elevation direction using the elevation focal point of the transducer as a virtual source element, in order to obtain dynamic focusing in the elevation plane.

A $0.1 \mathrm{~mm}$ point scatterer was mounted in an agar block and scanned in a water bath. The transducer is a 64 elements linear array with a pitch of $209 \mu \mathrm{m}$. The transducer height is $4 \mathrm{~mm}$ in the elevation plane and it is focused at $20 \mathrm{~mm}$ giving a F-number of 5 . The point scatterer was positioned $96 \mathrm{~mm}$ from the transducer surface. The transducer was translated in the elevation direction from -13 to $+13 \mathrm{~mm}$ over the scatterer at steps of $0.375 \mathrm{~mm}$. Each of the 70 planes is scanned using synthetic transmit aperture with 8 emissions. The beamformed RF lines from the planes are passed through a second beamformer, in which the fixed focal points in the elevation plane are treated as virtual sources of spherical waves. Synthetic aperture focusing is applied on them. The $-6 \mathrm{~dB}$ resolution in the elevation plane is increased from $7 \mathrm{~mm}$ to 2 $\mathrm{mm}$. This gives a uniform point spread function, since the resolution in the azimuthal plane is also $2 \mathrm{~mm}$.
\end{abstract}

\section{Introduction}

In the last years the interest in 3-D ultrasound imaging has been constantly increasing. However, due to technological limitations, there is only one real-time 3-D scanner [1], which uses 2-D matrix transducer arrays. Most other scanners employ conventional linear arrays to scan the volume of interest plane-by-plane, and then the information is reconstructed in a workstation. For a typical scan-depth of $15 \mathrm{~cm}$ and speed of sound $1500 \mathrm{~m} / \mathrm{s}$, the time for scanning a single plane consisting of 100 scan lines is $20 \mathrm{~ms}$. Because of the long acquisition time for a single plane, this method has a low frame rate. Another draw-back is the non-uniform resolution in the elevation and azimuth planes. The latter can be solved by using 1.5-D arrays, but the frame rate remains low.

The frame rate can be increased by employing a sparse transmit synthetic aperture as suggested in [2]. In this approach only a few emissions are used per plane. If only 5 emissions were used, the time for scanning the plane is reduced from $20 \mathrm{~ms}$ to $1 \mathrm{~ms}$, increasing the frame rate 20 times.

Previously a method for increasing the resolution of ultrasound images obtained by a fixed-focus transducer was suggested in [3]. In this approach the fixed focal point is treated as a virtual source of ultrasound, and the recorded RF lines are post focused to increase the resolution.

This paper suggests the combination of the two methods to improve both the frame rate and the resolution, since the linear array transducers are usually focused in the elevation plane. The planes are scanned one-by-one using synthetic transmit aperture focusing, and then the beamformed scan lines from the planes are refocused in the elevation plane to increase the resolution

The paper is organized as follows. Section 2 gives the theory behind the methods and how the two methods are combined. The results from simulations and measurements are given in Sections 3 and 4, respectively. Finally the conclusions are drawn in Section 5.

\section{Theoretical background}

The following sections give the theoretical background for obtaining images using a synthetic aperture imaging and for performing post focusing. 


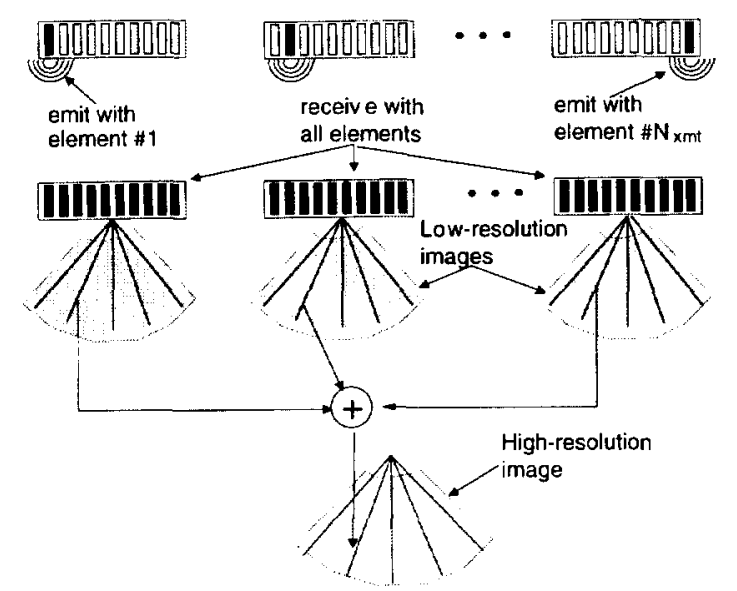

Figure 1: Synthetic transmit aperture focusing

\subsection{Synthetic transmit aperture}

When a single element of a linear array ultrasound transducer is excited, a spherical acoustic wave is created, provided that the element is small enough. The back scattered signal carries information from the whole region of investigation. In receive the RF lines in all directions are beamformed in parallel. Then another transmit element is excited and the process is repeated. The beamformed RF lines are summed after $N_{x m t}$ elements have been used in transmit. The beamforming process is shown in Fig. 1 and can be described as follows:

$$
\begin{aligned}
s_{l}(t) & =\sum_{i=1}^{N_{x m t}} \sum_{j=1}^{N_{x d c}} a_{l k j}(t) r_{k j}\left(t-\tau_{l k j}(t)\right), \quad l \in\left[1 \ldots N_{l}\right], \\
k & =f(i)
\end{aligned}
$$

where $l$ is the number of the scan line, $t$ is time relative to the trigger of the current transmit, $r_{k j}(t)$ is the signal received by the $j$ th element after transmitting with the $k$ th element. $a_{l k j}(t)$ and $\tau_{i k j}(t)$ are the applied apodization factor and delay, respectively. $N_{x d c}$ is the number of transducer elements, and $N_{x m t} \leq N_{x d c}$ is the number of emissions. The index of the transmitting element $k$ is related to the number of the current emission $i$ by a simple relation $f$. $k$ is equal to $i$, when $N_{x m t}=N_{x d c}$. Only some of the transducer elements are used in transmit if $N_{x m t}<N_{x d c}$, and a sparse transmit aperture is formed [2]. Because the delay $\tau_{l k j}(t)$ is applied only in receive, and is a function of time, the image is dynamically focused in transmit and receive assuming a linear and stationary propagation medium.

\subsection{Focusing using virtual source element}

In the elevation plane the transducers are either unfocused or have a fixed focus, and hereby the scanned image has a poor resolution in this plane.

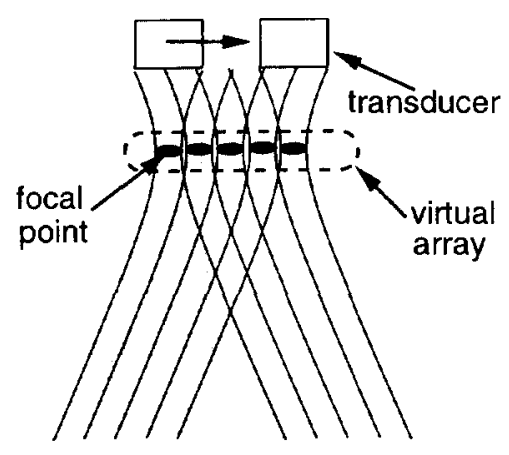

Figure 2: Forming a virtual array from the focal points of the scanned lines

Figure 2 shows a transducer in the elevation plane at several successive positions. The wave-front below the focal point can be considered as a spherical wave within a certain angle of divergence [3], and the focal point can be treated as a virtual source of ultrasound energy. The 3D volume is scanned by translating the transducer in the elevation direction in steps of $\Delta y$. The focal points lie on a line parallel to the transducer surface. The data can be considered as acquired by using one virtual element in transmit and receive.

Thus, synthetic aperture focusing can be applied on the beamformed RF lines from several emissions in order to increase the resolution in the elevation direction.

Let $n, 1 \leq n \leq N_{p}$, denote the position and $N_{p}$ be the number of several successive positions used for refocusing the data. The scan line $s_{l}(t)$ beamformed at position $n$ will be denoted as $s_{l}^{(n)}(t)$. The final lines in the volume $S_{l}(t)$ are beamformed according to:

$$
S_{l}(t)=\sum_{n=1}^{N_{p}} w_{n}(t) s_{l}^{(n)}\left(t-d_{n}(t)\right)
$$

where $w_{n}(t)$ is a weighting coefficient and $d_{n}(t)$ is the applied delay. The delay necessary to focus at a given distance $z$ ( $z \geq$ $f_{e z}$ ) is given by:

$$
\begin{aligned}
d_{n}(t) & =\frac{2}{c}\left(z-f_{e z}-\sqrt{\left(z-f_{e z}\right)^{2}+\left(\left(n-1-\frac{N_{p}-1}{2}\right) \Delta y\right)^{2}}\right) \\
t & =\frac{2 z}{c}
\end{aligned}
$$

where $c$ is the speed of sound and $f_{e z}$ is the distance to the elevation focus.

The best obtainable resolution in the elevation plane after post focusing is expected to be [4]:

$$
\delta y_{6 d B} \approx k \frac{0.41 \lambda}{\tan \frac{\theta}{2}}
$$




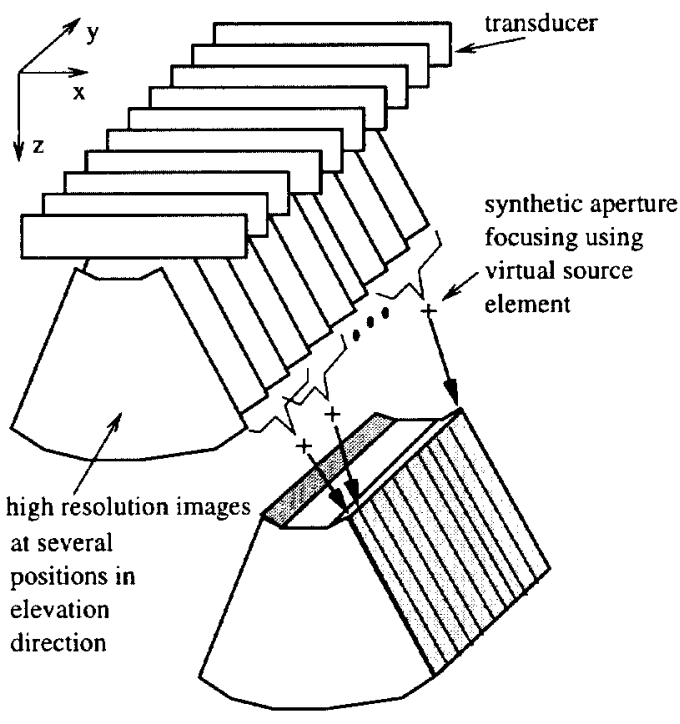

Figure 3: The beamforming stages for 3D focusing.

where $\lambda$ is the wavelength, and $\theta$ is the angle of divergence after the focal point. The variable $k(k \geq 1)$, is a coefficient depending on the apodization. For a rectangular apodization $k$ equals 1 and for Hanning apodization it equals 1.64 .

The angle of divergence can be approximated by [3]:

$$
\frac{\theta}{2} \approx \tan ^{-1} \frac{h}{2 f_{e z}},
$$

where $h$ is the size of the transducer in the elevation plane, and $f_{e z}$ is the distance to the fixed focus in the elevation plane. Substituting (5) in (4) gives:

$$
\delta y_{6 d B} \approx 0.82 \lambda k \frac{f_{e z}}{h}
$$

Equation (6) shows that the resolution is depth independent. However, this is true only if the number of the transducer positions is large enough to maintain the same Fnumber for the virtual array as a function of depth. For reallife applications the achievable resolution can be substantially smaller.

\subsection{Combining the two methods}

The whole process can be divided into two stages and is summarized in Fig. 3. In the first stage a high resolution image is created using only a few emissions, say $N_{x m t}=5$ as given in Fig. 1. This is repeated for several positions. Then the beamformed RF lines from these images are delayed, weighted, and summed a second time using (2) to form the final 3D volume. The considerations so far have been only for transducers translated in the $y$ direction. The synthetic aperture focusing is applicable for any kind of transducer motion (translation, rotation, or a free-hand scan), as long as the exact positions of the focal points are known.

\section{Simulations}
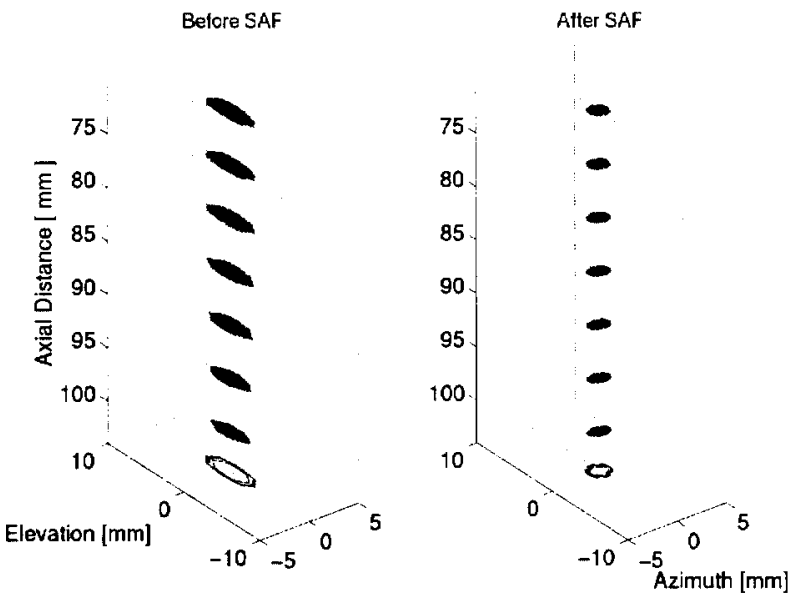

Figure 4: The 3-D point-spread function outlined at -10 dB.

The simulations were done using the program Field II [5]. The simulation parameters, given in Table 1, were chosen to match the parameters of the system used for the measurements.

\begin{tabular}{lccc}
\hline \hline Parameter name & Notation & Value & Unit \\
\hline Speed of sound & $c$ & 1480 & $\mathrm{~m} / \mathrm{s}$ \\
Sampling freq. & $f_{s}$ & 40 & $\mathrm{MHz}$ \\
Excitation freq. & $f_{0}$ & 5 & $\mathrm{MHz}$ \\
Wavelength & $\lambda$ & 296 & $\mu \mathrm{m}$ \\
-6 dB band-width & $B W$ & $4.875-10.125$ & $\mathrm{MHz}$ \\
Transducer pitch & $p$ & 209 & $\mu \mathrm{m}$ \\
Transducer kerf & $k e r f$ & 30 & $\mu \mathrm{m}$ \\
Number of elements & $N_{x d c}$ & 64 & - \\
Transducer height & $h$ & 4 & $\mathrm{~mm}$ \\
Elevation focus & $f_{e z}$ & 20 & $\mathrm{~mm}$ \\
\hline \hline
\end{tabular}

Table 1: Simulation parameters

Seven point scatterers lying at depths from 70 to $100 \mathrm{~mm}$ were simulated at 70 positions. The distance between every two positions in the elevation direction was $0.7 \mathrm{~mm}$. Figure 4 on the left shows the $-10 \mathrm{~dB}$ isosurfaces of the measured point-spread-functions (PSF). Then the beamformed scan lines were post-focused using $N_{p}=30$ planes to create one new plane. If a dynamic apodization is to be used, then the number of usable positions $N_{p}$ for depth $z$ from the real 


\begin{tabular}{cccc}
\hline \hline & & Before SAF & After SAF \\
Depth $[\mathrm{mm}]$ & $\delta x_{6 d B}[\mathrm{~mm}]$ & $\delta y_{6 d B}[\mathrm{~mm}]$ & $\delta y_{6 d B}[\mathrm{~mm}]$ \\
\hline 70 & 1.44 & 4.78 & 1.72 \\
75 & 1.54 & 5.16 & 1.72 \\
80 & 1.65 & 5.48 & 1.72 \\
85 & 1.75 & 5.80 & 1.85 \\
90 & 1.85 & 6.18 & 1.85 \\
95 & 1.96 & 6.56 & 1.85 \\
100 & 2.06 & 6.75 & 1.97 \\
\hline \hline
\end{tabular}

Table 2: The resolution at $-6 \mathrm{~dB}$ as a function of depth.

transducer can be determined by:

$$
N_{p}=\left\lfloor 2 h \frac{z-f_{e z}}{f_{e z}} \frac{1}{\Delta y}\right\rfloor,
$$

Figure 4 on the right shows the PSF after the post focusing was applied. Table 2 shows the $-6 \mathrm{~dB}$ resolution in the azimuth and the elevation planes. The lateral size of the PSF in the azimuth plane increases linearly with depth:

$$
\delta x_{6 d b}=z \sin \phi_{6 d B},
$$

where $\phi_{6 d B}$ is the angular size of the PSF in polar coordinates.

The $\delta y_{6 d B}$ prior to the synthetic aperture focusing also increases almost linearly with depth, which shows that the beam is diverging with a certain angle as shown in Fig. 2. After applying the synthetic aperture focusing $\delta y_{6 d B}$ becomes almost constant as predicted by (6). A Hann window was used for $w_{n}$, and this gives $k \approx 1.6$. Substituting $h=4 \mathrm{~mm}, f_{e z}=20$ $\mathrm{mm}$, and $\lambda=0.296 \mathrm{~mm}$, gives $\delta y_{6 d B} \approx 1.87$.

\section{Measurements}

The measurements were done using the department's off-line experimental system XTRA [6]. The parameters of the system are the same as the ones used in the simulations and are given in Table 1.

In [3] it is argued that due to the narrow angle of divergence after the focal point, the grating lobes are greatly suppressed. Therefore it is possible to traverse the elevation direction at steps $\Delta y$ bigger than one wavelength $\lambda$. Two experiments were conducted:

1. A point scatterer mounted in an agar block $96 \mathrm{~mm}$ away from the transducer was scanned, at step $\Delta y=375 \mu \mathrm{m}$. The diameter of the point scatterer was $100 \mu \mathrm{m}$.

2. A wire phantom was scanned at steps of $\Delta y=700 \mu \mathrm{m}$. The wires were positioned at depths from 45 to $105 \mathrm{~mm}$, $20 \mathrm{~mm}$ apart. At every depth there were two wires, perpendicular to each other. The diameter of the wires was $0.5 \mathrm{~mm}$.

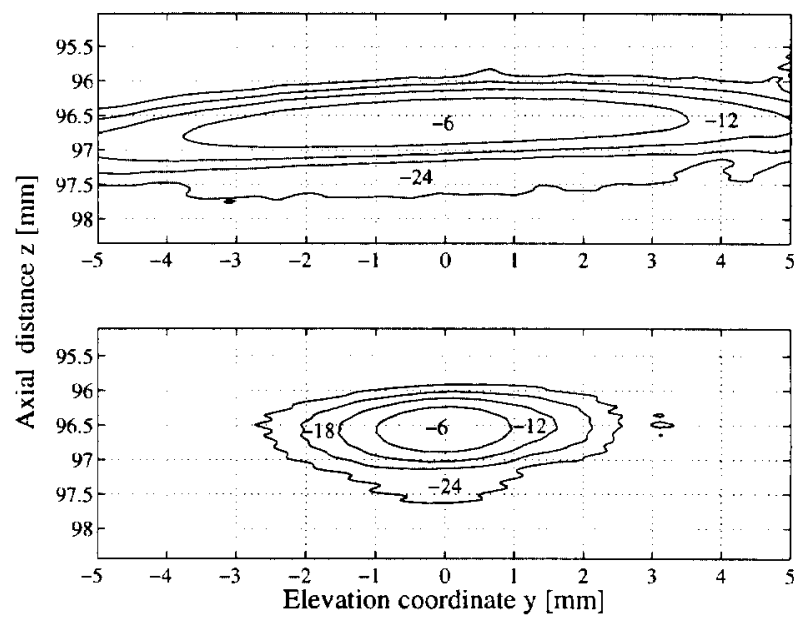

Figure 5: PSF in the elevation plane: (top) before and (bottom) after synthetic aperture focusing. The innermost contour is at level of $-6 \mathrm{~dB}$, and the difference between the contours is also $6 \mathrm{~dB}$.

The first experiment was conducted to verify the resolution achieved in the simulations. The goal of the second experiment was to verify that the resolutions in the elevation and azimuthal planes are comparable in size.

Using only a few emissions per plane corresponds to using a sparse transmit aperture. The use of wires as a phantom gives a good signal-to-noise ratio (compared to the $0.1 \mathrm{~mm}$ point scatterer) necessary to evaluate the level of the associated grating lobes. The SNR was further increased by using 11 elements in transmit to create a spherical wave instead of 1 as described in [2].

Figure 5 shows the PSF of the point scatterer in the elevation plane. The contours are drawn at levels $6 \mathrm{~dB}$ apart. Sixty planes $\left(N_{p}=60\right)$ were used in the post-focusing. This maintains the same size of the virtual array as the one in the simulations. The achieved resolution at $-6 \mathrm{~dB}$ is $2 \mathrm{~mm}$ and is comparable with the resolution obtained in the simulations.

Figure 6 shows a $-10 \mathrm{~dB}$ outline of the wire phantom. The image was beantformed using $N_{x m t}=8$ emissions per plane. The post focusing was performed using $N_{p}=21$ planes for each new. The image shows that the resolution in the elevation and azimuth planes are of comparable size.

One of the problems of the approach are the grating lobes. They can be caused by two factors: (a) using only a few emissions per plane and (b) using a large step $\Delta y$ between two planes.

The step $\Delta y$ must not exceed the size of the PSF at the elevation focus, in order not to get image with discontinuities. For a larger step $\Delta y$, a transducer with a higher F-number must be used. Such transducers have a smaller angle of divergence $\theta$ and therefore the level of the grating lobes in the elevation direction is greatly suppressed. However, this is not 

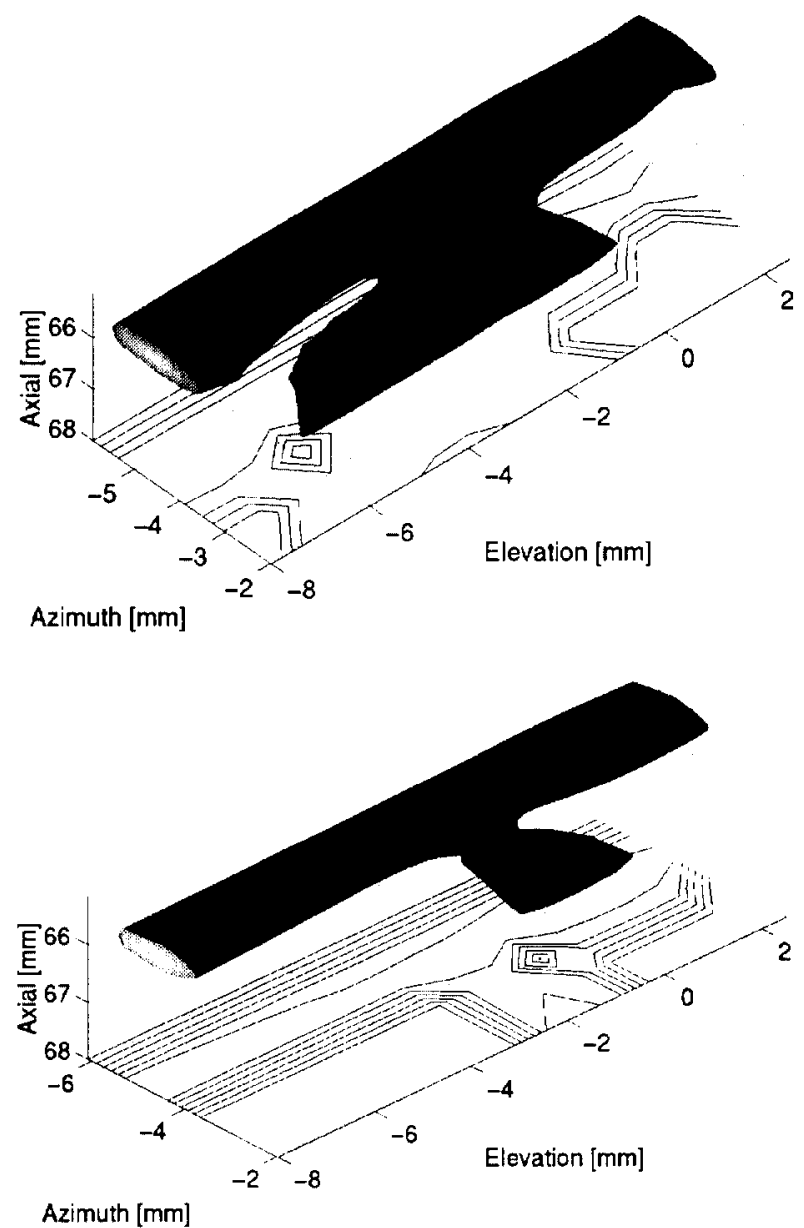

Figure 6: Outline at $-10 \mathrm{~dB}$ of the wire phantom: (top) before, and (bottom) after post focusing.

the case in the azimuth plane. Table 3 shows the compromise between the level of the grating lobes and the maximum available number of planes for the post focusing. The figures are derived for speed of sound $c=1540 \mathrm{~m} / \mathrm{s}$ and scan depth $\max (z)=15 \mathrm{~cm}$. The table shows, that the larger the number of emissions per position $N_{x m t}$ is, the larger the step $\delta y$ must be, in order to maintain the number of volumes per second constant. This requires the use of a transducer which is not strongly focused in the elevation plane.

\section{Conclusions}

An approach for 3-D scanning using synthetic transmit aperture in the azimuth plane followed by synthetic aperture post focusing in the elevation plane was presented. The acquisition is done plane by plane using a conventional linear array transducer. The obtained resolution in the elevation plane is

\begin{tabular}{cccc}
\hline \hline$N_{x m t}$ & Position & Level & $\max \left(N_{p}\right)^{1}$ \\
\hline 64 & NA & NA & 7 \\
13 & \pm 17 & $-51.0 \mathrm{~dB}$ & 38 \\
8 & \pm 10 & $-44.5 \mathrm{~dB}$ & 62 \\
5 & \pm 7 & $-41.3 \mathrm{~dB}$ & 100 \\
\hline \hline
\end{tabular}

Table 3: Grating lobes in the azimuth plane.

$\approx 2 \mathrm{~mm}$, and is comparable to the one in the azimuth plane Up to 10 volumes per second can be scanned if only 8 emissions per plane are used, and each volume contains 62 planes. This makes the approach a feasible alternative for real-time 3 D scanning.

\section{Acknowledgements}

This work was supported by grant 9700883 and 9700563 from the Danish Science Foundation and by B-K Medical $\mathrm{A} / \mathrm{S}$.

Some of the measured data were supplied by Søren Pihl Rybro, Peter Foged Christensen, and Madalina Breten.

The measurement system was built by Søren Kragh Jespersen as part of his Ph.D. study.

\section{References}

[1] O. T. von Ramm, S. W. Smith, and Henry G. Pavy. Highspeed ultrasound volumetric imaging system - Part II: Parallel processing and image display. IEEE Trans. $\mathrm{Ul}$ trason., Ferroelec., Freq. Contr., 38:109-115, 1991.

[2] G. R. Lockwood, J. R. Talman, and S. S. Brunke. Realtime 3-D ultrasound imaging using sparse synthetic aperture beamforming. IEEE Trans. Ultrason., Ferroelec., Freq. Contr., 45:980-987, 1998.

[3] C. H. Frazier and W. D. O'Brien. Synthetic aperture techniques with a virtual source element. IEEE Trans. Ultrason., Ferroelec., Freq. Contr., 45:196-207, 1998.

[4] C. Passmann and H. Ermert. A 100-MHz ultrasound imaging system for dermatologic and ophthalmologic diagnostics. IEEE Trans. Ultrason., Ferroelec., Freq. Contr., 43:545-552, 1996.

[5] J. A. Jensen. Field: A program for simulating ultrasound systems. Med. Biol. Eng. Comp., 10th Nordic-Baltic Conference on Biomedical Imaging, Vol. 4, Supplement 1, Part 1:351-353, 1996b.

[6] S. K. Jespersen, J. E. Wilhjelm, and H. Sillesen. Multiangle compound imaging. Uttrason. Imaging, 20:81$102,1998$. 